\title{
Filtration-Assisted Magnetic Micropatterning of Bacterial Cellulose
}

\author{
Tsunehisa KImURA, ${ }^{1, \dagger, \dagger \dagger}$ Tetsuya KAMIOKA,${ }^{1}$ and Shigenori KugA ${ }^{2}$ \\ ${ }^{1}$ Department of Applied Chemistry, Tokyo Metropolitan University, 1-1 Minami-ohsawa, Hachioji 192-0397, Japan \\ ${ }^{2}$ Department of Biomaterials Sciences, The University of Tokyo, 1-1-1 Yayoi, Bunkyo-ku, Tokyo 113-8657, Japan
}

(Received December 12, 2006; Accepted July 31, 2007; Published September 14, 2007)

KEY WORDS Magnetic Patterning / Bacterial Cellulose / Suspension / [doi:10.1295/polymj.PJ2006189]

Micropatterning of particles has attracted considerable attention in various areas of science and technology. A number of techniques, including photo or electron-beam/ion-beam lithography and microcontact printing techniques, ${ }^{1-5}$ have been used to provide two-dimensional patterning. In a previous paper, ${ }^{6}$ we reported a patterning of diamagnetic particles using a modulated magnetic field. A field modulator consisting of alternating sheets of aluminum and iron of $300 \mu \mathrm{m}$ thick was inserted into a homogeneous magnetic field to modulate the magnetic field over the modulator surface. Diamagnetic particles including polymer spheres ${ }^{6}$ and cells ${ }^{7}$ suspended in a medium were attracted to the location where the field strength is weak, resulting in a pattern following the pattern of the field modulation.

Since the field modulation persists only in a distance of the order of the modulator width from the modulator surface, particles that are floating beyond this persistent distance cannot be patterned. A solution to this problem is to evaporate the medium. With decrease in the level of the liquid medium, the particles reach within the persistent distance to be trapped. However, the evaporation causes the change in composition of the medium, leading to coagulation of particles, damages to cells, etc. in some cases.

In this note bacterial cellulose (BC) was chosen for the demonstration purpose. Because $\mathrm{BC}$ produced stays floated in a medium, the conventional magnetic patterning technique is not applied successfully. We combined the magnetic technique with filtration to enable micropatterning of $\mathrm{BC}$.

Acetobacter xylinum, JCM 10150 (Japan Collection of Microorganisms) was used to produce BC. A BC particle obtained by static culture was immersed in the phosphate buffer to obtain a suspension of acetobacterium. The suspension and an aqueous solution of $\mathrm{Mn}$ (II)EDTA $(0.1 \mathrm{M})$ were mixed at $2: 3$ by volume to make the medium paramagnetic. $2 \mathrm{~mL}$ of the prepared suspension was poured into the plastic dish and subjected to cultivation at $30^{\circ} \mathrm{C}$ for $3.5 \mathrm{~h}$ with or without magnetic field. During this cultivation time, BC is formed.

A plastic dish $(40 \mathrm{~mm}$ diameter) with a hole $(15 \mathrm{~mm})$ cut in the bottom was used. The hole is covered with a Millipore filter (Omnipore JGWP02500, polytetrafluoroethylene, $25 \mathrm{~mm}$ diameter, $0.2 \mu \mathrm{m}$ pore size, $80 \mu \mathrm{m}$ thick) glued to the bottom. This plastic dish was placed on a field modulator ${ }^{6}$ (alternating layer of aluminum and iron sheet of $300 \mu \mathrm{m}$ ) with the Millipore filter being in contact with the modulator surface. Whole of the dish and the modulator was tightly fixed between two poles pieces of an electromagnet generating a horizontal magnetic field of $1 \mathrm{~T}$.

The experimental setup with a field modulator is shematically shown in Figure 1. In the case that a horizontal magnetic field is applied to the modulator, the magnetic flux density is reduced on the iron layers, resulting in formation of minima on the iron layers. The depth of the minima depends on the distance from the surface of the modulator. The modulation persists approximately over the distance comparable to the iron and aluminum layer thickness.

The magnetic force acting on a suspended particle is proportional to $V \Delta \chi \nabla B^{2}$, where $\Delta \chi=\chi_{\mathrm{p}}-\chi_{\mathrm{m}}$ is the difference between the magnetic susceptibility of the particles $\chi_{\mathrm{p}}$ and that of the medium $\chi_{\mathrm{m}}$. Both $\chi_{\mathrm{p}}$ and $\chi_{\mathrm{m}}$ are negative because $\mathrm{BC}$ and the medium are diamagnetic; $V$ is the size of the particle; $\nabla B^{2}$ is the magnetic force field. The $\nabla B^{2}(=2 B \mathrm{~d} B / \mathrm{d} x)$ value, which is estimated using the modulation profile shown in Figure 1, is approximately $2,000 \mathrm{~T}^{2} / \mathrm{m}$. This value is comparable to that necessary for a water droplet to levitate in the air $\left(1,400 \mathrm{~T}^{2} / \mathrm{m}\right)$. If $\Delta \chi<0$, the particles are attracted to the minima of the field. The difference $\Delta \chi$ between $\mathrm{BC}$ and the medium is so small

\footnotetext{
${ }^{\dagger}$ To whom correspondence should be addressed (Tel: +81-75-753-6246, Fax: +81-75-753-6300, E-mail: tkimura@kais.kyoto-u.ac.jp).

${ }^{\dagger}$ Present address: Division of Forest and Biomaterials Science, Kyoto University, Kitashirakawa Oiwake-cho, Kyoto 606-8502, Japan.
} 


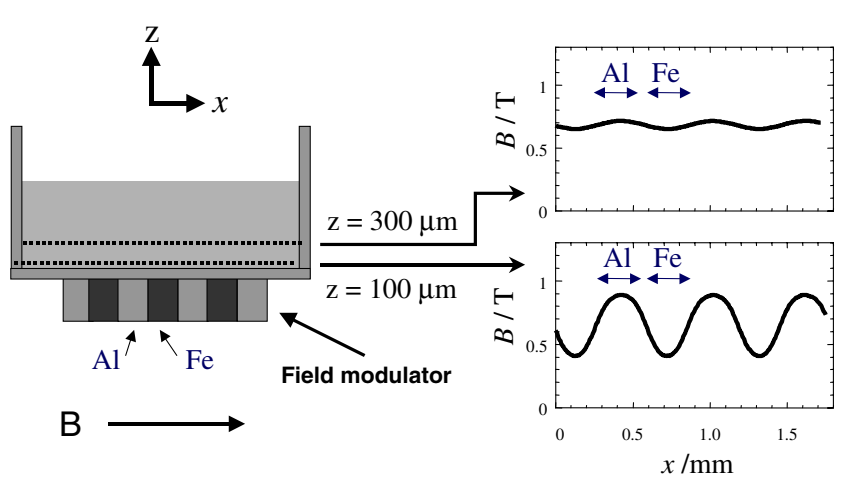

Figure 1. Schematic diagram of magnetic patterning by means of a field modulator (left). A uniform magnetic field B is modulated over the surface of the modulator composed of alternating aluminum and iron layers. The modulation persists over the distance comparable to the aluminum and iron layer thickness (here $300 \mu \mathrm{m}$ is assumed) (right). Particles are trapped on the iron layers if $\Delta \chi<0$.

that the magnetic force generated is not strong enough to trap BC. In order to enhance the magnetic force, a paramagnetic compound is often added to the medium. In the present study, Mn(II)-EDTA (paramagnetic) was used.

The speed of sedimentation of $\mathrm{BC}$ suspended in the medium is very slow or BC even does not sink. Therefore, it would take a long time or is impossible to achieve the patterning because the magnetic force is effective only in the vicinity of the bottom that is in contact with the modulator. In order to accelerate the patterning, removal of the suspending medium by filtration is useful. Schematic diagram of this method is shown in Figure 2. Due to the capillary force acting on a filtered liquid that is in the narrow space between the top surface of the modulator and the bottom surface of the Millipore filter, the medium coming out through the Millipore filter gradually leaks away. It took about $3 \mathrm{~h}$ for the completion of filtration.

In Figure 3a, BC suspended in the plastic dish is

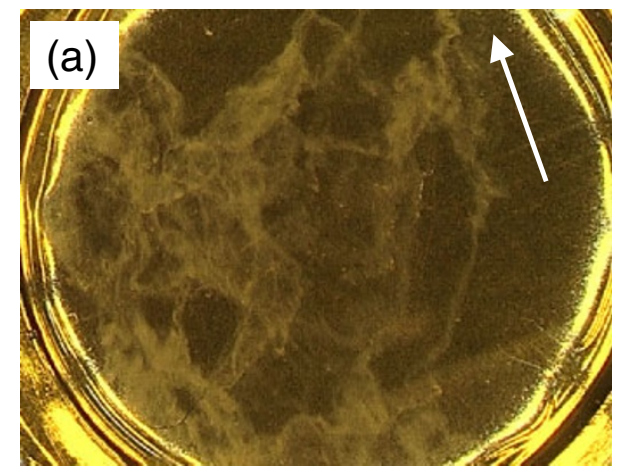

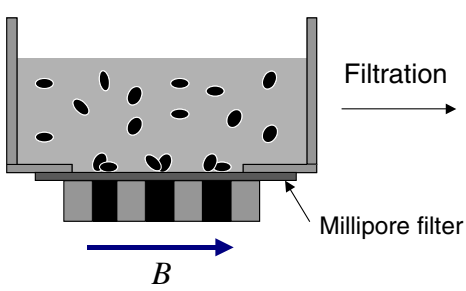

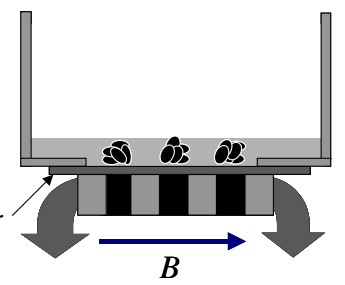

Figure 2. Schematic diagram showing the magnetic patterning assisted by filtration. The medium passes through the Millipore filter and is removed from the plastic dish. Upon decrease of the liquid level, particles floating in the medium reaches the filter surface where they are captured by the modulated field.

shown. The bottom of the dish is covered with a Kapton film that is in contact with the field modulator subjected to the magnetic field. Only a very small portion of $\mathrm{BC}$ that happens to be close to the substrate surface is trapped to form a very blurred line pattern. However, most of the $\mathrm{BC}$ remains floated in the cultivation medium without being trapped even after a prolong time. On the other hand, if we use a Millipore filter instead of a Kapton film, the cultivation medium is allowed to leak away slowly. As a result, the BC that is unable to pass through the filter is trapped during the filtration of the medium to form the line pattern as shown in Figure $3 \mathrm{~b}$. Because the pattern is not formed when the magnetic field is not applied to the modulator, the pattern formation is attributed to the magnetic effect.

There are some factors that could affect the formation of pattern. If the amount of the BC floating in the medium exceeds the amount acceptable to the field minima, the imperfect localization of $\mathrm{BC}$ would take place: the extra amount of $\mathrm{BC}$ would be located between lines. The velocity profile of the medium in the vicinity of the substrate surface could affect the formation of pattern. Because the medium is paramagnetic, the medium tends to flow toward the surface of

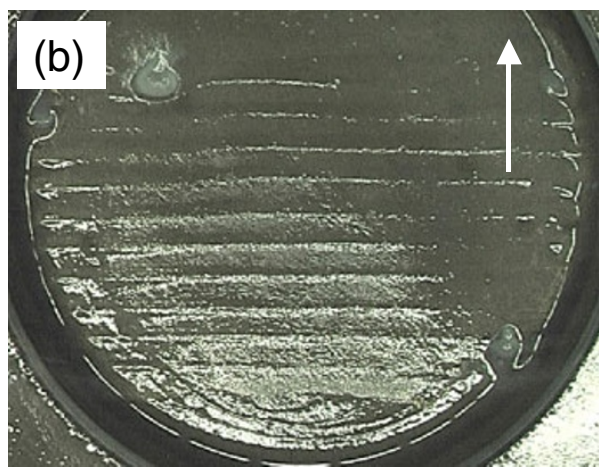

Figure 3. Optical micrographs of BC subjected to modulated magnetic field. The direction of the applied magnetic field is indicated by an arrow. (a) A Kapton film is used for substrate. Most of the suspended BC remains floated; only a very small portion is forming a line pattern on the substrate. (b) A Millipore filter is used for substrate. A line pattern is clearly formed after the cultivation medium is removed by filtration. The distance between the lines is $c a$. $600 \mu \mathrm{m}$. 
aluminum layer where the field is more intense. The particle is attracted toward the surface of the iron layer. This attractive force should overcome the flow of the medium that goes opposite to the motion of the particle. The size of $\mathrm{BC}$ is also an important factor. Even if a single BC fiber is not large enough, a strong enough force could act if BC fibers are highly flocculated.

In conclusion, we reported a novel method of micropatterning of floating particles in a suspension: the filtration and the magnetic trap by the modulated field were combined. This technique was applied to the patterning of $\mathrm{BC}$ to obtain a line pattern, but it may be also useful for patterning of other small particles in a suspension that is too slow to reach the trapping zone by sedimentation.

Acknowledgment. This work was partially supported by Grant-in-Aid for Scientific Research on Pri- ority Area 'Innovative utilization of strong magnetic fields' (Area 767, No. 15085207) from MEXT of Japan.

\section{REFERENCES}

1. M. Geissler and Y. Xia, Adv. Mater., 16, 1249 (2004).

2. C. K. Yee, M. L. Amweg, and A. N. Parikh, Adv. Mater., 16, 1184 (2004).

3. M. H. V. Werts, M. Lambert, J.-P. Bourgoin, and M. Brust, Nano Lett., 2, 43 (2002).

4. M. G. Ancona, S. E. Kooi, W. Kruppa, A. W. Snow, E. E. Foos, L. J. Whitman, D. Park, and L. Shirey, Nano Lett., 3, 135 (2003).

5. H. O. Jacobs and G. M. Whitesides, Science, 291, 1763 (2001).

6. T. Kimura, M. Yamato, and A. Nara, Langmuir, 20, 572 (2004).

7. T. Kimura, Y. Sato, F. Kimura, M. Iwasaka, and S. Ueno, Langmuir, 21, 830 (2005). 